\title{
Coinfecção tuberculose/HIV: perfil sociodemográfico e saúde de usuários de um centro especializado
}

Tuberculosis/HIV co-infection: sociodemographic and health profile of users of a specialized center Coinfección tuberculosis/VIH: perfil sociodemográfico y salud de usuarios de un centro especializado

Shyrlaine Honda Bastos ${ }^{1}$ io https://orcid.org/0000-0003-1674-0823

Monica Taminato ${ }^{1}$ is https://orcid.org/0000-0003-3307-2644

Mariza Vono Tancredi ${ }^{1}$ io https://orcid.org/0000-0002-3371-8700

Carla Gianna Luppi ${ }^{1}$ io https://orcid.org/0000-0001-9183-8594

Lúcia Yasuko Izumi Nichiata1 io https://orcid.org/0000-0001-6515-4404

Paula Hino ${ }^{1}$ io https://orcid.org/0000-0002-1408-196X

Bastos SH, Taminato M, Tancredi Como citar: Nichiata LY, Hino P. Coinfecção tuberculose/HIV: perfil sociodemográfico e saúde de usuários de um centro especializado. Acta Paul Enferm. 2020;33:APE20190051.

DOI

http://dx.doi.org/10.37689/actaape/2020A000515

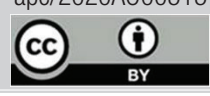

Descritores Sindrome da imunodeficiência adquirida; Enfermagem em saúde comunitária; Doenças crônicas; Serviços de saúde

Keywords

Tuberculosis; Acquired immunodeficiency syndrome; Community health nursing; Chronic disease; Health services

Descriptores Tuberculosis: Síndrome de inmunodeficiencia adquirida; Enfermería em salud comunitaria; Enfermedad crónica; Servicios de salud

\section{Submetido 8 de Março de 2019 \\ Aceito \\ 18 de Dezembro de 2020}

\section{Autor correspondente}

Shyrlaine Honda Bastos Email: shyrlainehonda@gmail.com

\section{Resumo}

Objetivo: Descrever algumas características sociodemográficas e de saúde de pessoas que apresentaram a coinfecção tuberculose e vírus da imunodeficiência humana notificada em um centro especializado do município de São Paulo.

Métodos: Estudo descritivo de casos de coinfecção tuberculose/HIV notificados em um Centro de Referência e Treinamento de Doenças Sexualmente Transmissíveis e Aids do município de São Paulo, entre 2007 e 2016. A fonte de informações foi 0 Sistema de Notificação e Acompanhamento dos Casos de Tuberculose - TBWEB.

Resultados: Foram notificados 745 casos de coinfecção, 76,6\% eram do sexo masculino, 67,8\% encontravamse na faixa etária de 30 a 49 anos e 62,6\% possuíam oito anos ou mais de escolaridade. Em relação ao desfecho do tratamento da tuberculose, $46,3 \%$ dos casos evoluíram para cura e 13,3\% foram a óbito. Ao comparar os períodos de notificação de 2007-2011 e 2012-2016, pôde-se observar que ocorreu uma redução percentual de 43,0\% no número de casos notificados e 76,6\% na frequência de evolução para óbito.

Conclusão: 0 conhecimento da situação epidemiológica da coinfecção tuberculose/HIV possibilita fornecer subsídios para repensar as atividades do cuidado em saúde e para 0 aprimoramento de políticas públicas.

\section{Abstract}

Objective: To describe some sociodemographic features and health of individuals who present tuberculosis, and HIV co-infection of a specialized center located in the municipality of Sao Paulo.

Methods: This was a descriptive study of tuberculosis/HIV co-infection reported in a referral and training center of sexual transmissible diseases and AIDS in the municipality of Sao Paulo, conducted between 2007 and 2016. The source of information was a notification system and follow-up of tuberculosis cases.

Results: We notified 745 cases of co-infection, $76.6 \%$ were men, $67.8 \%$ were aged between 30 to 49 years, and $62.6 \%$ had 8 years or more of formal education. In relation to end of treatment of tuberculosis, $46.3 \%$ of cases evolved cure and $13.3 \%$ were death. The comparison of periods of notification from 2007-2011 and 2012-2016 can be observed by the occurrence of percentage reduction of $43.0 \%$ in the number of reported cases, and $76.6 \%$ in frequency of evolution of death.

Conclusion: The knowledge of the epidemiological situation of tuberculosis/HIV co-infection enables the provision of subsides to rethink the activities of health care and to enhance public policies.

\section{Resumen}

Objetivo: Describir algunas características sociodemográficas y de salud de personas que presentaron la coinfección tuberculosis y virus de la inmunodeficiencia humana notificada en un centro especializado del municipio de São Paulo.

'Escola Paulista de Enfermagem, Universidade Federal de São Paulo, São Paulo, SP, Brasil.

Conflitos de interesse: Embora Hino P e Taminato M são Editoras Associadas da Acta Paulista de Enfermagem, ambas não tiveram acesso ao processo de avaliação do manuscrito. 
Métodos: Estudio descriptivo de casos de coinfección tuberculosis/VIH notificados en un Centro de Referencia y Capacitación de Enfermedades de Transmisión Sexual y SIDA del municipio de São Paulo, entre 2007 y 2016. La fuente de información fue el Sistema de Notificación y Seguimiento de Casos de Tuberculosis (TBWEB).

Resultados: Se notificaron 745 casos de coinfección, el 76,6 \% del sexo masculino, el 67,8 \% del grupo de edad de 30 a 49 años y el 62,6 \% con ocho 0 más años de escolaridad. Con relación al resultado del tratamiento de la tuberculosis, el 46,3\% de los casos se recuperó y el 13,9 \% falleció. Al comparar los períodos de notificación 2007-2011 y 2012-2016, puede observarse que se redujo un 43,0 \% la cantidad de casos notificados y un 76,6 \% la frecuencia de muerte.

Conclusión: El conocimiento sobre la situación epidemiológica de la coinfección tuberculosis/VIH permite proporcionar subsidios para repensar las actividades de cuidado de la salud y para la mejora de políticas públicas.

\section{Introdução}

A ocorrência da tuberculose (TB) ativa em pessoas que vivem com o vírus da imunodeficiência humana (HIV) representa um desafio e impacta a sua mortalidade. As pessoas infectadas pelo HIV apresentam 26 vezes mais chance de desenvolver TB ativa quando comparadas à população geral. ${ }^{(1)}$ Além disso, é frequente a descoberta da infecção em ocasião do diagnóstico de TB. ${ }^{(2)}$ Portanto, o HIV representa um problema de saúde pública e tem contribuído para o aumento de casos de $\mathrm{TB}$, refletindo-se nas taxas de cura, abandono e mortalidade..$^{(3-4)}$

A TB ocupa a nona causa de morte em todo o mundo, acometendo principalmente pessoas que vivem com HIV e aids (PVHA). Em 2017 estimou-se no mundo a ocorrência de 6.708 .123 notificações de TB, e, destas, 6,9\% apresentaram coinfecção pelo HIV. ${ }^{(5)}$ Observou-se em 2015 que o Brasil ocupava a $20^{a}$ posição de países prioritários para a abordagem da TB e a $19^{a}$ posição no que se refere à coinfecção $\mathrm{TB} / \mathrm{HIV} .^{(6)}$

No Brasil, a taxa de coinfecção TB/HIV calculada para o ano de 2016 foi de $9,7 \%$, variando conforme região do país, de $7,9 \%$ na região Nordeste a $17,3 \%$ na região Sul. ${ }^{(7)}$ Observou-se que no país, em 2016, 73,2\% dos casos novos de TB realizaram testagem para o HIV, tendo a regiáo Sul apresentado melhor desempenho (acima de 80\%), seguida da Sudeste $(78,7 \%)$, Centro-Oeste $(67,1 \%)$, Norte $(66,8 \%)$ e Nordeste $(62,1 \%)$. Embora haja a recomendaçáo para que todos os casos diagnosticados por TB sejam testados para o HIV, visto que o diagnóstico do HIV possibilita a introdução precoce da terapia antirretroviral (TARV), há importante diferença entre as regióes do país. ${ }^{(8)}$
Dada a estreita relação entre TB e HIV, ressalta-se que as duas enfermidades devem ser abordadas de forma integrada pelos programas de controle, de forma que estes propiciem a construção de uma proposta articulada e de acordo com as particularidades das pessoas que apresentam a coinfecção. ${ }^{(9)}$ Há possibilidade de obtenção de resultados favoráveis de controle de ambas com a busca de casos, de forma a agilizar o diagnóstico precoce e garantir a adesão ao tratamento. ${ }^{(10)}$

$\mathrm{O}$ tratamento de pessoas que apresentam a coinfecção TB/HIV é mais complexo, pois exige a adesão a dois esquemas terapêuticos: o da TB deve ser realizado em um período de no mínimo 6 meses, enquanto o do HIV é ininterrupto. Desta forma, a quantidade elevada de medicamentos a serem ingeridos diariamente e a maior probabilidade de ocorrência de eventos adversos representam um desafio para a adesão ao tratamento. ${ }^{(11)}$

Um estudo transversal realizado em um hospital público no Ceará com pessoas que apresentavam a coinfecção TB/HIV evidenciou a adesão ao tratamento comprometida em parcela significativa dos entrevistados, o que impacta de forma negativa a condição de saúde destas pessoas. O seguimento correto do tratamento tanto da TB quanto do HIV é fundamental para garantir que se tenha uma boa qualidade de vida e prolongamento da vida, com diminuição das taxas de abandono do tratamento e de mortalidade. ${ }^{(11)}$

Assim, para o controle da coinfecção TB/HIV é necessário que as ações sejam articuladas de modo a oferecerem a testagem do HIV para todas as pessoas com diagnóstico de TB, o tratamento da TB ativa e da infecção latente, bem como iniciar oportunamente a TARV. ${ }^{(12,13)}$ Considerando a relevância da temática, esta pesquisa apresentou a seguinte questão norteadora: "qual é o perfil de pessoas que apre- 
sentaram a coinfecção TB/HIV considerando um período de 10 anos?"

Frente à situação apresentada, o estudo teve como objetivo descrever algumas características sociodemográficas e de saúde de pessoas que apresentaram a coinfecção TB/HIV notificadas em um Centro de Referência e Treinamento de Doenças Sexualmente Transmissíveis e Aids do município de São Paulo.

\section{Métodos}

Trata-se de um estudo descritivo dos usuários que apresentaram coinfecçáo TB/HIV atendidos em um centro especializado do município de São Paulo. A população do estudo foi composta de todos os casos com diagnóstico de TB e HIV, com idade igual ou superior a 18 anos, que apresentavam diagnóstico de TB e HIV em um período de 10 anos (janeiro de 2007 a dezembro de 2016). Foram excluídos os casos que realizaram transferência do acompanhamento para outro serviço e os registros duplicados no sistema, ou seja, aqueles que apresentaram os mesmos dados de identificação e data de diagnóstico.

Em março de 2018 foram coletados dados referentes ao perfil sociodemográfico e de saúde de pessoas com a coinfecção TB/HIV no Sistema de Notificação e Acompanhamento dos Casos de Tuberculose - TBWEB, da Divisáo de Epidemiologia do referido serviço de saúde.

As variáveis do estudo foram: sexo, faixa etária, raça/cor, escolaridade, ano de notificação da $\mathrm{TB}$, tipo de caso, encerramento do tratamento da TB, forma clínica, classificação, evolução, óbito e período de notificação (2007/2011 e 2012/2016).

As informaçôes foram armazenadas em planilha eletrônica do software Microsoft Excel ${ }^{\circledR}$ e analisadas por meio do software SPSS (Statistical Package for the Social Ciência, versão 19). Foram utilizadas ferramentas da estatística descritiva (frequência absoluta e relativa) e analítica ( $\mathrm{X}^{2}$ de Person, nível de significância de 5\%).

O projeto de pesquisa foi aprovado pelos Comitês de Ética em Pesquisa de uma universidade pública e pelo serviço de saúde onde ocorreu a coleta de dados (pareceres número 2.408 .003 e 2.476.518).

\section{Resultados}

Durante o período do estudo (2007 a 2016), foram notificados 745 casos de coinfecção TB/HIV. Deste total, $76,6 \%$ eram do sexo masculino, com predomínio da faixa etária de 30 a 49 anos $(67,8 \%)$, cor branca $(60,8 \%)$ e escolaridade de oito a 11 anos de estudo (46,0\%) (Tabela 1).

Tabela 1. Distribuição dos casos notificados por tuberculose e HIV segundo algumas características sociodemográficas. Centro de Referência de DST/Aids

\begin{tabular}{|c|c|c|}
\hline Variáveis & $\mathrm{n}(\%)$ & $p$-value \\
\hline Sexo & & 0,323 \\
\hline Masculino & $571(76,6)$ & \\
\hline Feminino & $174(23,4)$ & \\
\hline Faixa etária & & 0,823 \\
\hline$\leq 19$ anos & $12(1,6)$ & \\
\hline 20 a 29 anos & $99(13,3)$ & \\
\hline 30 a 39 anos & $262(35,2)$ & \\
\hline 40 a 49 anos & $243(32,6)$ & \\
\hline 50 a 59 anos & $107(14,4)$ & \\
\hline 60 a 69 anos & $19(2,5)$ & \\
\hline 70 a 79 anos & $3(0,4)$ & \\
\hline Raça/cor & & 0,739 \\
\hline Branco & $453(60,8)$ & \\
\hline Pardo & $170(22,8)$ & \\
\hline Preto & $95(12,8)$ & \\
\hline Ignorado e em branco & $16(2,1)$ & \\
\hline Amarelo & $9(1,2)$ & \\
\hline Indígena & $2(0,3)$ & \\
\hline Escolaridade (anos) & & 0,541 \\
\hline 0 a 3 & $46(6,2)$ & \\
\hline 4 a 7 & 195(26,2) & \\
\hline 8 a 11 & $343(46,0)$ & \\
\hline 12 ou mais & $124(16,6)$ & \\
\hline Ignorado & $37(5,0)$ & \\
\hline Total & $745(100,0)$ & \\
\hline
\end{tabular}

Fonte: Sistema de Notificação e Acompanhamento dos Casos de Tuberculose - TB Web.

A tabela 2 apresenta informaçóes dos casos que apresentaram a coinfecção TB/HIV de 2007 a 2016. Em relação ao tipo de caso, a maioria era caso novo $(63,3 \%)$, destaca-se que em todo o período apenas $11(1,5 \%)$ dos casos foram retratamento após falência/resistência. Dos 745 casos notificados no período, $46,3 \%$ apresentaram encerramento por cura, $22,8 \%$ por abandono e $13,3 \%$ evoluíram para óbito. No que diz respeito à classificação, notou-se a forma pulmonar exclusiva em 46,0\% dos casos e $25,0 \%$ apresentaram a forma mista (pulmonar e extrapulmonar). 
Tabela 2. Casos de pessoas que vivem com HIV segundo o tipo de caso de tuberculose, encerramento e forma clínica. Centro de Referência e Treinamento DST/Aids

\begin{tabular}{lcc}
\hline Variáveis & $\mathrm{n}(\%)$ & $p$-value \\
\hline Tipo de caso & & 0,572 \\
$\quad$ Novo & $474(63,6)$ & \\
Recidiva & $131(17,6)$ & \\
Retratamento/Abandono & $129(17,3)$ & \\
$\quad$ Retratamento após falência/resistência & $11(1,5)$ & \\
Encerramento & & $p<0,01$ \\
$\quad$ Cura & $345(46,3)$ & \\
Abandono & $170(22,8)$ & \\
Mudança de diagnóstico & $108(14,5)$ & \\
Óbito & $99(13,3)$ & \\
Falência/Resistência & $12(1,6)$ & \\
Transferência & $8(1,1)$ & \\
Em branco & $3(0,4)$ & \\
Forma clínica & & 0,169 \\
Pulmonar & $529(71,0)$ & \\
Ganglionar periérica & $56(7,5)$ & \\
Múltiplos órgãos & $49(6,6)$ & \\
Meníngea & $41(5,5)$ & \\
Pleural & $33(4,4)$ & \\
$\quad$ Outras formas & $37(4,9)$ & \\
Classificação & & \\
Pulmonar & 34179 \\
Pulmonar + extrapulmonar & $186(25,0)$ & \\
Extrapulmonar & $167(22,4)$ & \\
Disseminada & $49(6,6)$ & \\
Total & $745(100,0)$ & \\
\hline
\end{tabular}

Fonte: Sistema de Notificação e Acompanhamento dos Casos de Tuberculose - TB Web.

A tabela 3 apresenta o número de casos notificados com coinfecção TB/HIV e a evolução para óbito segundo os períodos de notificação. A maioria dos casos com coinfecção TB/HIV foram notificados entre os anos de 2007 e 2011 , sendo que neste período foram notificados 476 casos, enquanto no período subsequente (2012/2016) observaram-se 269 casos notificados, ou seja, ocorreu uma redução percentual de 43,0\%. A evolução para óbito dos casos notificados também foi maior de 2007 a 2011: $63,9 \%$, com redução no período subsequente para $36,1 \%$. A redução percentual da frequência de óbitos entre esses dois períodos foi de 76,6\%.

Tabela 3. Casos de coinfecção TB/HIV segundo evolução para óbito e período de notificação. Centro de Referência e Treinamento DST/Aids

\begin{tabular}{lccc}
\hline Período de notificação & $\begin{array}{c}\text { Óbito } \\
\text { Sim }(\mathbf{n}=99) \\
\mathbf{n}(\%)\end{array}$ & $\begin{array}{c}\text { Total } \\
\mathbf{n}(\%)\end{array}$ & p-value \\
\hline 2007 a 2011 & $75(75,8)$ & $476(63,9)$ & 0,042 \\
2012 a 2016 & $24(24,2)$ & $269(36,1)$ & \\
Total & $99(100,0)$ & $745(100,0)$ & \\
\hline
\end{tabular}

Fonte: Sistema de Notificação e Acompanhamento dos Casos de Tuberculose - TB Web.

\section{Discussão}

A análise das características sociodemográficas e de saúde de pessoas com coinfecção TB/HIV notificadas no Sistema de Acompanhamento dos Casos de Tuberculose mostrou que há diferenças em termos percentuais de algumas variáveis.

A maioria dos casos notificados foi do sexo masculino, faixa etária de 30 a 49 anos e com oito anos ou mais de escolaridade. Destaca-se que no período analisado ocorreu uma importante redução do número de casos de coinfecção TB/HIV notificados, acompanhada da redução da frequência de evolução de óbitos ao longo do período observado.

O predomínio de casos em pessoas do sexo masculino $(76,6 \%)$ corroborou achados na literatura. ${ }^{(10-19)}$ Uma possível causa para o predomínio do sexo masculino refere-se ao aumento da notificação dos casos de aids entre este grupo e a baixa detecção e notificação da aids entre mulheres adultas no período de 2006 a 2017, exceto entre as mulheres idosas. ${ }^{(20)}$ Uma Revisão Sistemática (RS) realizada em países de baixa renda revelou que outra possível razão é que os homens possuem uma prevalência mais alta de TB, pois retardam a procura por atendimento, permanecendo mais tempo infectados em relação às mulheres, o que se reflete no diagnóstico e tratamento oportuno. ${ }^{(21)}$

No presente estudo foi encontrado predomínio de casos na faixa etária de 30 a 49 anos. Ainda que variável na literatura nacional ${ }^{(14,15,17,19,22,23)}$ e internacional, ${ }^{(24,25)}$ ressalta-se que essa faixa etária abrange a fase em que as pessoas se encontram economicamente ativas, fato que pode impactar diretamente a renda familiar e a economia do país. No entanto, justamente no Brasil, onde as taxas de desemprego têm sido altas, é possível verificar o impacto sobre as pessoas que acabam por desenvolver a TB e HIV. Um estudo transversal realizado no estado do Ceará sobre a adesão aos antirretrovirais revelou elevado índice de desemprego entre os coinfectados, que, concomitantemente, eram também os indivíduos que apresentaram baixa adesão aos antirretrovirais em comparação aos que se encontravam empregados. ${ }^{(11)}$ Um estudo realizado em Portugal com o objetivo de identificar os fatores sociodemográficos 
relacionados à incidência de $\mathrm{TB}$, revelou que, além do HIV, o desemprego foi um agravante para a incidência da TB, confirmando que a TB é uma doença socialmente determinada. ${ }^{(26)}$

A variável raça-cor que se encontrou com maior frequência neste estudo foi branca, o que corrobora outras investigaçóes. ${ }^{(12,27)}$ Por outro lado, pesquisas apontam o contrário, evidenciando predomínio da raça/cor preta e parda. ${ }^{(10,13,14,19,28,29)}$ Um estudo realizado em São Paulo nos anos de 2006 a 2013, que buscou analisar o perfil epidemiológico dos casos novos de TB, revelou maior incidência de TB em indivíduos indígenas e pretos. ${ }^{(23)}$ Pesquisa realizada com pessoas coinfectadas diagnosticadas entre janeiro de 2008 e julho de 2016 em um serviço de saúde do município do Rio de Janeiro revelou maior atraso da assistência à saúde às pessoas de raça/cor negra ou parda, tanto em relação à busca pelo atendimento quando pela sua demora. ${ }^{(30)}$ Todavia, como a infecção atinge indivíduos independentemente da raça-cor e de várias etnias, essa pode ser uma análise subjetiva.

No presente estudo foi encontrada elevada frequência de pessoas que apresentaram a coinfecção TB/HIV que possuíam oito anos de estudo ou mais, diferentemente da literatura nacional, que apontou menor escolaridade dos indivíduos coinfectados, muitos deles analfabetos ou que tinham cursado apenas o ensino fundamental. ${ }^{(10,12,13,17,19,28,29)} \mathrm{Um}$ estudo de caráter observacional realizado na China mostrou que $56 \%$ dos coinfectados possuíam apenas o nível primário e, dessa forma, menos percepção da própria saúde. ${ }^{(31)}$ No entanto, esse resultado pode ser decorrente das características da população atendida nesse serviço de referência.

A literatura nacional e internacional revelam que as pessoas com coinfecção TB/HIV apresentam maior risco de evoluírem para óbito quando comparadas àquelas que têm apenas $\mathrm{TB} \cdot{ }^{(3,22)} \mathrm{A}$ coinfecção TB/HIV torna a pessoa mais suscetível ao estado avançado da aids, o que aumenta o risco de morte, e é ainda uma das principais causas de morte entre os adultos internados em todo o mundo, de acordo com RS realizada com estudos de 42 países. ${ }^{(32)}$ Esses dados reforçam a relevância do tema nos esforços das políticas públicas para diminuir a coinfecção TB/HIV.
Segundo dados do Ministério da Saúde, ${ }^{(29)}$ foram identificados 6.501 casos novos de coinfecção em 2016 no Brasil, e, destes, 78,4\% apresentavam a forma clínica pulmonar. No mesmo ano, de acordo com a Organizaçáo Mundial de Saúde (OMS), dos 5,4 milhóes de casos novos de TB pulmonar no mundo, $57 \%$ tiveram confirmação bacteriológica. ${ }^{(5)}$ Os achados nacionais ${ }^{(12,14,15,19,28)}$ mostraram que a forma clínica pulmonar é a mais prevalente nos casos de coinfecção. Alguns estudos ${ }^{(10,28)}$ também demonstraram o aumento da forma clínica extrapulmonar. No entanto, é sabido que o diagnóstico de TB em PVHA pode ser dificultado devido à maior chance de exames falso-negativos, acrescida da possibilidade da ocorrência de formas extrapulmonares e/ou imagem pulmonar atípica em exame radiológico. ${ }^{(15)}$ Uma RS mostrou uma outra questão, a de existirem poucos estudos sobre a relação do HIV com a TB extrapulmonar, sendo necessários mais estudos que possam enriquecer a discussão. ${ }^{(33)}$

Da mesma forma, observa-se que o diagnóstico precoce da TB e o tratamento oportuno das pessoas coinfectadas associados às melhores condiçôes de vida e açôes intersetoriais podem ter um impacto no controle da TB. ${ }^{(34)} \mathrm{Um}$ estudo nacional que analisou os casos novos de TB nos anos de 1991 a 2010 na cidade de Olinda - PE revelou que os exames de baciloscopia de escarro e o teste de esfregaço foram subutilizados, e que, juntamente com a falta de informação sobre o resultado do teste anti-HIV, impedem o conhecimento da real situação da coinfecção TB/HIV. ${ }^{(28)}$ No âmbito internacional, achados de um estudo realizado na Nigéria revelou um alto índice de incidência de TB nos indivíduos que eram soropositivos e que iriam iniciar a TARV, mostrando a necessidade de realizaçáo precoce do teste de TB nos indivíduos com HIV para melhor manejo do tratamento. ${ }^{(35)}$

A letalidade elevada neste grupo específico pode estar relacionada ao diagnóstico tardio, à baixa adesão ao tratamento e ao desenvolvimento de resistência ao tratamento. ${ }^{(1)}$ Observou-se queda da mortalidade ao longo do período analisado. Para explicar essa redução é importante considerar que várias ações foram organizadas e implementadas ao longo do tempo, tais como as voltadas para a investigação das duas enfermidades. Portanto, o 
diagnóstico precoce e início imediato do tratamento da TB é fundamental, pois o óbito em casos de coinfecção TB/HIV ocorre principalmente nos primeiros dois meses do tratamento da $\mathrm{TB}$, razão pela qual o diagnóstico deve ser realizado o mais precocemente possível, assim como é indispensável a testagem para HIV na totalidade dos casos diagnosticados com TB. ${ }^{(10,13)}$

A adesão ao tratamento dessas duas enfermidades deve ser permeada pelo estabelecimento de vínculo entre usuário e profissional de saúde, por um ambiente acolhedor e favorável à construção de possibilidades que minimizem o sofrimento. Portanto, lidar com pessoas coinfectadas pela TB/HIV impóe que os profissionais repensem as práticas em saúde de modo ampliado e humanizado, valorizando a escuta dos significados e as percepçóes destas pessoas frente à situação de saúde e tratamentos a serem seguidos e, desta forma, prestando assistência de qualidade voltada para as demandas e necessidades de saúde. $^{(4)}$

Este estudo apresenta algumas limitaçóes, tais como o uso de dados secundários, que envolve a questão do não preenchimento de algumas variáveis que constam na ficha de notificação compulsória, e a coleta de dados ter sido feita apenas no TB - WEB, o que impossibilitou o acesso a outras informaçóes relevantes, como, por exemplo, o ano de notificação do HIV e uso de TARV. Além disso, a investigação foi realizada em apenas um centro de referência DST/Aids, fato que revela uma realidade local e não pode ser generalizada.

\section{Conclusão}

Esse estudo permitiu conhecer a situação epidemiológica de pessoas com a coinfecção TB/HIV notificadas em um centro especializado por um período de dez anos. Os resultados mostraram diferenças percentuais de algumas variáveis e que dada à estreita relação entre ambas as doenças, podem contribuir para a organização das açóes de cuidado de pessoas vivendo com HIV, assim como a implementação de políticas públicas que envolvem o controle tanto da TB quando do HIV.

\section{Agradecimentos}

Ao Conselho Nacional de Ciência e Tecnologia (CNPq; Bolsa de Iniciação Científica para Shyrlaine Honda Bastos).

\section{Colaborações}

Bastos SH, Taminato M, Tancredi MV, Luppi CG, Nichiata LYI e Hino P colaboraram com a concepção e desenvolvimento do projeto, análise dos dados, redação do artigo, revisão crítica relevante do conteúdo intelectual e aprovação final da versão a ser publicada.

\section{Referências}

1. World Health Organization (WHO). Global Tuberculosis Report, 2015. Geneva: WHO; 2015 [Internet]. 2015 [cited 2018 Apr 30]:78. Available from: http://apps.who.int/iris/ bitstream/10665/191102/1/9789241565059_eng.pdf

2. Brasil. Ministério da Saúde. Recomendações para o manejo da coinfecção TB-HIV em serviços de atenção especializada a pessoas vivendo com HIV/AIDS [Internet]. Brasília (DF): Ministério da Saúde; 2013. [citado 2018 Mai 5]. Disponivel em: http://bvsms.saude.gov.br/ bvs/publicacoes/recomendacoes_manejo_coinfeccao_tb_hiv.pdf

3. Velásquez GE, Cegielski JP, Murray MB, Yagui MJA, Asencios LL, Bayona JN, et al. Impact of HIV on mortality among pacients treated for tuberculosis in Lima, Peru: a prospective cohort study. BMC Infect Dis. 2016;16(45):1-11.

4. Silva JB, Cardoso GC, Ruffino-Netto A, Kritski AL. Os significados da comorbidade para os pacientes vivendo com TB/HIV: repercussões no tratamento. Rev Saúde Coletiva. 2015;25(1):209-29.

5. World Health Organization (WHO). Global Tuberculosis Report, 2018 [Internet]. Geneva: WHO; 2017. [cited 2019 Sep 23]: Available from: https://www.who.int/tb/publications/global_report/en/

6. Brasil. Ministério da Saúde. Secretaria de Vigilância em Saúde. Departamento de Vigilância das Doenças Transmissíveis. Plano Nacional pelo fim da tuberculose [Internet]. Brasília (DF): Ministério da Saúde; 2017. [citado 2018 Mai 5]. Disponível em: http://bvsms.saude. gov.br/bvs/publicacoes/brasil_livre_tuberculose_plano_nacional.pdf

7. Brasil. Ministério da Saúde. Perspectivas brasileiras para o fim da tuberculose como problema de saúde pública. Bol Epidemiol. 2016; 47(13):1-15.

8. Brasil. Ministério da Saúde. Secretaria de Vigilância em Saúde. Indicadores prioritários para o monitoramento do Plano Nacional pelo Fim da Tuberculose como Problema de Saúde Pública no Brasil [Internet]. Brasília (DF): Ministério da Saúde; 2017 [citado 2018 Mai 5];48(8). Disponivel em: http://portalarquivos2.saude.gov.br/images/ pdf/2017/marco/23/2017-V-48-N-8-Indicadores-priorit--rios-para-0monitoramento-do-Plano-Nacional-pelo-Fim-da-Tuberculose-comoProblema-de-Sa--de-P--blica-no-Brasil.pdf 
9. Magnabosco GT, Lopes LM, Andrade RL, Brunello ME, Monroe AA, Villa TC. Tuberculosis control in people living with HIV/AIDS. Rev Lat Am Enfermagem. 2016;24(0):e2798.

10. Torrens A, Bartholomay P, Silva S, Khogali M, Verdonck K, Bissell K. HIV testing, antiretroviral therapy, and treatment outcomes in new cases of tuberculosis in Brazil, 2011. Rev Panam Salud Publica. 2016;39(1):26-31.

11. Lemos LA, Fiuza ML, Reis RK, Ferrer AC, Gir E, Galvão MT. Adherence to antiretrovirals in people coinfected with the human immunodeficiency virus and tuberculosis. Rev Lat Am Enfermagem. 2016;24(0):e2691.

12. Castrighini CC, Reis RK, Neves LA, Galvão MT, Gir E. Prevalence and epidemiological aspects of HIV/tuberculosis coinfection. Rev Enferm UERJ. 2017;25:e17432.

13. Rebouças MC, Silva MO, Haguihara T, Brites C, Netto EM. Tuberculosis incidence among people living with HIV/AIDS with virological failure of antiretroviral therapy in Salvador, Bahia, Brazil. Braz J Infect Dis. 2017;21(5):562-6.

14. Magno ED, Saraceni V, Souza AB, Magno RD, Saraiva MD, BührerSékula S. Factors associated with TB/HIV coinfection: evidence from notification data in the State of Amazonas, Brazil, 2001-2012. Cad Saude Publica. 2017;33(5):e00019315.

15. Barbosa IR, Costa IC. Estudo epidemiológico da coinfecção tuberculoseHIV no nordeste do Brasil. Rev Patol Trop. 2014;43(1):27-38.

16. Costa KB, Silva CE, Martins AF. Clinical and epidemiological characteristics of patients with tuberculosis in the city with the highest incidence of the disease in Brazil. Clin Biomed Res. 2014;34(1):40 6.

17. Lima MD, Martins-Melo FR, Heukelbach J, Alencar CH, Boigny RN, Ramos AN. Mortality related to tuberculosis-HIV/AIDS co-infection in Brazil, 2000-2011: epidemiological patterns and time trends. Cad Saude Publica. 2016;32(10):e00026715.

18. Teklu AM, Nega A, Mamuye AT, Sitotaw Y, Kassa D, Mesfin G, et al. Factors associated with mortality of TB/HIV co-infected patients in Ethiopia. Ethiop J Health Sci. 2017;27 Suppl 1:29-38.

19. Prado TN, Rajan JV, Miranda AE, Dias ED, Cosme LB, Possuelo LG, et al. Clinical and epidemiological characteristics associated with unfavorable tuberculosis treatment outcomes in TB-HIV co-infected patients in Brazil: a hierarchical polytomous analysis. Braz J Infect Dis. 2017;21(2):162-70.

20. Brasil. Ministério da Saúde. Boletim Epidemiológico: HIV AIDS 2018 [Internet]. Brasília (DF): Ministério da Saúde; 2018 [citado 2019 Set 27]. Disponível em: http://www.aids.gov.br/pt-br/pub/2018/boletimepidemiologico-hivaids-2018.

21. Horton KC, MacPherson P, Houben RM, White RG, Corbett EL. Sex Differences in Tuberculosis Burden and Notifications in Low- and Middle-Income Countries: A Systematic Review and Meta-analysis. PLoS Med. 2016;13(9):e1002119.
22. Gaspar RS, Nunes N, Nunes M, Rodrigues VP. Temporal analysis of reported cases of tuberculosis and of tuberculosis-HIV co-infection in Brazil between 2002 and 2012. J Bras Pneumol. 2016;42(6):416-22.

23. Pinto PF, Silveira C, Rujula MJ, Chiaravalloti F, Ribeiro MC. Perfil epidemiológico da tuberculose no município de São Paulo de 2006 a 2013. Rev Bras Epidemiol. 2017;20(3):549-57.

24. Korzeniewska-Koseła M, Kuś J, Lewandowska K, Siemion-Szcześniak I. Tuberculosis in homeless persons in Poland. Przegl Epidemiol. 2015;69(3):445-51.

25. Amadou ML, Abdoulaye 0, Amadou 0, Biraïma A, Kadri S, Amoussa AA, et al. Epidemiological, clinical and evolutionary profile of patients with tuberculosis at the Regional Hospital of Maradi, Republic of the Niger. Pan Afr Med J. 2019;33:120.

26. Sousa P, Oliveira A, Gomes M, Gaio AR, Duarte R. Longitudinal clustering of tuberculosis incidence and predictors for the time profiles: the impact of HIV. Int J Tuberc Lung Dis. 2016;20(8):1027-32.

27. Peruhype RC, Acosta LM, Ruffino Netto A, Oliveira MM, Palha PF. The distribution of tuberculosis in Porto Alegre: analysis of the magnitude and tuberculosis-HIV coinfection]. Rev Esc Enferm USP. 2014;48(6):1035-43.

28. Silva AP, Souza WV, Albuquerque MF. Two decades of tuberculosis in a city in Northeastern Brazil: advances and challenges in time and space. Rev Soc Bras Med Trop. 2016;49(2):211-21.

29. Brasil. Ministério da Saúde. Secretaria de Vigilância em Saúde. Coinfecção TB-HIV no Brasil: Panorama epidemiológico e atividades colaborativas [Internet]. Brasília (DF): Ministério da Saúde; 2017 [citado 2018 Mai 5]. Disponível em: http://www.aids.gov.br/pt-br/pub/2017/coinfeccao-tb-hivno-brasil-panorama-epidemiologico-e-atividades-colaborativas-2017.

30. Nogueira BM, Rolla VC, Akrami KM, Kiene SM. Factors associated with tuberculosis treatment delay in patients co-infected with HIV in a high prevalence area in Brazil. PLoS One. 2018;13(4):e0195409.

31. Zhu Y, Wu J, Feng X, Chen H, Lu H, Chen L, et al. Patient characteristics and perceived health status of individuals with HIV and tuberculosis coinfection in Guangxi, China. Medicine (Baltimore). 2017;96(14):e6475.

32. Ford N, Matteelli A, Shubber Z, Hermans S, Meintjes G, Grinsztejn B, et al. TB as a cause of hospitalization and in-hospital mortality among people living with HIV worldwide: a systematic review and metaanalysis. J Int AIDS Soc. 2016;19(1):20714.

33. Shivakoti R, Sharma D, Mamoon G, Pham K. Association of HIV infection with extrapulmonary tuberculosis: a systematic review. Infection. 2017;45(1):11-21

34. Duarte R, Lönnroth K, Carvalho C, Lima F, Carvalho AC, MuñozTorrico M, et al. Tuberculosis, social determinants and co-morbidities (including HIV). Pulmonology. 2018;24(2):115-9.

35. Musa BM, Musa B, Muhammed H, Ibrahim N, Musa AG. Incidence of tuberculosis and immunological profile of TB/HIV co-infected patients in Nigeria. Ann Thorac Med. 2015;10(3):185-92. 